\title{
Qualidade do uso de medicamentos por idosos: uma revisão dos métodos de avaliação disponíveis
}

Quality assessment of drug use in the elderly: a review of available evaluation methods

Andréia Quei roz Ribei ro 1

Cristiana Martins do Co uto Araújo 1

Francisco de Assis Acurcio 1

Sérgia Maria Starling Magalhães 1

Flávio Chaimowicz 2

\footnotetext{
1 Departamento de Fa rmácia Social, Faculdade de Fa rmácia da UFMG. Campus Pampulha. Av. Presidente Antônio Carlos, 6.627, 31270-901, Belo Horizon te MG. aribeiro@farmacia.ufmgbr 2 Departamento de Cínica Médica, Faculd ade de Medicina da UFMG.
}

\begin{abstract}
The article presents different methods for the assessment of the pharmacotherapy appropriateness for the elderly, after the litera ture review within 1990 and 2003. In the 1990's a great va riety of meth ods was cre a ted and they are classified according to the criteria use in implicit, explicit and the ones that blend both implicit and explicit criteria. The advantages and disadvantages of each method are presented and it is observed that methods that use a criteria combination allow a better assessment, since they incorpora te a gre a ter number of evolved elements in the drug use process. The suitability and adaptation of these methods to the Brazilian reality and their incorporation in the evaluative practices in health can constitute an essential step to the rational drug use in the country.

Key words Elderly, Pharmacotherapy, In appropriate drug use, Assessment
\end{abstract}

Resumo $O$ artigo apresenta diferentes métodos de avaliação da adequação da terapia farmacológica pa ra idosos, a pa rtir da revisão da litera tu ra no período de 1990 a 2003. Na década de 1990, foi criada uma variedade de métodos os quais são dassificados, de aco rdo com a utilização de critérios, em implícitos, explícitos e aqueles que combinam ambos. São apresentadas as vantagens $e$ desvantagens de cada método e ob serva-se que os métodos que utilizam combinação de critérios permitem uma melhor avaliação, uma vez que incorpo ram um maior número de elemen tos envolvidos no processo de utilização de medicamentos. A adequação ou adaptação destes métodos à realidade brasilei ra e a inco rpo ração dos mesmos às prá ticas avaliativas em saúde pod em se constituir em um passo fundamental na promoção do uso racional de medicamentos no país.

Palavras-chave Idosos, Farmacoterapia, Uso inadequado de medicamentos, Avaliação 


\section{Introdução}

O envelhecimen to da população brasileira deverá se acelerar substantivamen te a partir de 2005, quando as coortes de nascidos nos períodos de elevada fecundidade e baixa mortalidade das décadas de 1940 a 1960 começa rem a alcançar os 65 anos. Estima-se que a proporção de idosos saltará dos atuais $5,1 \%$ para $14,2 \%$ em apenas 50 anos, fenômeno cuja magnitude e velocidade será igualada por menos de 10 países em todo o mundo (Chaimowicz, 1997). Esse fenômeno reperc ute nas diferen tes esferas das estrutu ras social, econômica, sanitária, política e cultural da sociedade, uma vez que os idosos, freqüen temen te portadores de múltiplas doenças crônico-degenerativas, possu em demandas específicas para obtenção de adequadas condições de vida (Siqueira et al., 2002). Tais demandas fazem do envel hecimen to tema emergen te de investigação nas distintas áreas de conhecimen to, tais como a Farmacoepidemiologia. Esta tem como foco o estudo da distribuição e dos determinantes dos acontecimentos relacionados com os fármacos nas populações e a aplicação desses estudos a uma terapêutica farmacológica eficaz (Last, 1989).

Com as alterações fisiológicas e patológicas relacionadas ao envelhecimento, há um aum ento dos riscos associados ao uso de fármacos. Den tre os fatores que con tribu em para este aumento destacam-se o comprometimento da função renal para a depuração de fárm acos que são primariamente exc ret ados pelos rins; a redução do flu xo sanguíneo e do processo de bi otra $\mathrm{n}$ sformação hep á tica; o aumen to da gordu ra corpórea, o que re sulta no aumen to do volume de distri buição de fárm acos lipossolúveis. Além disso, alterações da sensibilidade de receptores e as modificações da resposta dos sistemas fisiológicos com prom eti dos por doenças podem alterar a ação dos fármacos (Katzung, 2002). Permean do todos estes fatores, de st aca-se a influência da qualidade da prescrição e do uso de medicamen tos neste grupo.

Nesta perspectiva, nos países de senvolvidos, a partir de meados da década de 1980 e, principalmente, da década de 1990, a preocupação com os efeitos prejudiciais do uso de medicamentos por idosos impulsionou prescritores, farmacêuticos e pesquisadores a desenvo verem e aplicarem diversos métodos e instrumen tos para iden tificar padrões inadequados de prescrição e probl emas farm aco terapêuticos envo lven do este grupo populacional.
No Brasil, estudos e aplicações sobre esta temática são escassos. Informações sobre o padrão de uso de med i ca m en tos en tre idosos brasileiros são provenien tes de estudos conduzidos nos municípios do Rio de Janeiro, Fortaleza e nas cidades de Cam po Belo e Bambuí, em Minas Gerais (Rozenfeld, 1997; Miralles \& Kimberl in, 1998; Mosegui et al., 1999; Chaimowicz et al., 2000; Coelho Filho et al., 2004). Apenas dois destes estu dos investigaram aspectos relaci on ados à qualid ade da farmacoterapia. No estudo realizado no Rio de Janei ro verificou-se que $17 \%$ dos medicamentos utilizados eram inadequados para uso por idosos. Além disso, $14 \%$ das idosas faziam uso de medicamentos redundantes e $16 \%$ estavam ex postas às principais interações medicamentosas e, portanto, sujeitas às conseqüências desses even tos (Mosegui et al. , 1999). Em Fortaleza, quase $20 \%$ do s idosos usavam pelo menos um medicamen to considerado inadequado para esta faixa etária (Coelho Filho et al., 2004). Essa carência de informações caracteriza uma das dificuldades para a implementação de uma política de assistência farmacêutica adequada à realidade brasilei rae, conseqüentemente, para a melh oria da qualidade da atenção à saúde do idoso no país.

O objetivo do presente artigo é revisar os principais instrumentos utilizados no âmbi to in ternacional para avaliar a adequação da farmacoterapia em idosos, bem como discutir a aplicação de stes instrum en tos à luz da realidade brasileira.

\section{Métodos}

Foi realizada busca na base de dados Medline por trabalhos referen tes a critérios de definição de uso inadequado de medicamen tos por idosos no período de 1990 a 2003. As palavras-chave utilizadas foram older adults, elderly, prescription, criteria, inappropriate, a ppropriateness.

A combinação destes descritores identificou um total de 106 documentos distintos, den tre os quais 46 foram descart ados por se referirem a critérios de adequação de prescrição sobre uma única classe de medicamentos ou s obre terapias não farmacológicas. Assim, restaram 60 documentos potenciais para análise. De s te univers o, quatro documen tos se referi a $m$ à implementação de programas computacionais para aprim orar o processo de prescrição e 29 artigos não propunham critérios ou métodos específicos. Estes artigos foram excluídos, 
restando 27 para análise. Nos documentos selecion ados para análise foi realizada, po steri ormente, uma busca manual das referências listadas sobre o tema em questão. Tais referências foram obtidas em bibliotecas ou por meio de con $t$ a to com os autores re s pon $s$ á veis pelas mesmas. Seguindo esse procedimen to, obteve-se a de s c rição de seis crit érios que foram discutidos nessa revisão. Vale ressaltar que apenas os estudos que tra tavam da descrição dos critérios foram referen ci ado s, uma vez que este era o obj etivo da pre s en te revisão.

\section{Resultados}

A qualidade do uso de medicamentos pode ser abordada sob diferen tes aspectos, tais como a prática de polifarmácia, a subutilização de fár$\mathrm{m}$ acos necessários e o uso inadequ ado de especialidades terapêuticas. Conceitualmente, um med ica m en to é con si derado inadequ ado qu a ndo os ris cos de seu uso su peram seus benefícios (Beers et al., 1991).

$\mathrm{Na}$ atualidade, há uma variedade de métodos destinados à avaliação da adequação farmaco terapêutica para idosos. De uma maneira geral, estes métodos se baseiam em critérios implícitos, explícitos, ou na combinação de ambos. Os métodos implícitos caracterizam-se por revisão clínica dos medicamentos em uso levando em conta as prá ticas con s i deradas adequ adas nas revisões de literatura médica sobre as doenças específicas apresentadas pelos pacientes. Entret a n to, não têm uma preocupação de definir ou pad ronizar critérios e carecem de uma estrutu ra de revisão baseada em con s en so. Os métodos explícitos, mais limitados no que se refere à adequação clínica, geralmen te são baseados em métodos de con s enso e inclu em a utilização de listas con tendo medicamentos a serem evitados por idosos (Shelton et al., 2000).

Pa ra fins desse arti go, s erão apre s en t ados os métodos mais importantes, no sentido de serem pioneiros, serem referência para adaptações e serem mais amplamente utilizados.

\section{Métodos implícitos}

Es tes métodos caracterizam-se por revisões terapêuticas específicas para cada indivíduo e não estabelecem critérios de avaliação. Um exemplo é o Medication Reduction Project (MEDRED) desenvolvido em Dakota do Sul
(Estados Unidos) em 1993. Trata-se de um programa de base comunitária fundamentado na farmácia clínica e com intervenções edu ca tivas para reduzir a polifarmácia em idosos (Schrader et al., 1996). Conduzido em diferen tes et apas, o MEDRED possui cinco metas, quais sejam, reduzir o número de medicamentos utilizados; a justar as doses; aumentar a adesão à terapia; identificar os impactos social, funcional e econômico de um tratamento medicamen toso; e incentivar o uso de alternativas não farmacológicas quando dinicam en teindicado.

Na con dução do MEDRED, a etapa de revisão dos medicamen tos foi realizada por um farmacêuti co especialista em geriatria. Esta revisão ocorreu de maneira individualizada para 83 idosos da comunidade, com en foque sobre os seguintes aspectos: con hecimen to do indivíduo sobre o medicamen to e a doença em tra tam en to, existência de um diagnóstico que justificasse a indicação do medicamento, efetividade e adequação do fárm aco e da dose utilizada, existência de condições clínicas rel aci on adas ao uso de medicamen tos (ou reação adversa) e a adesão (Sch rader et al., 1996).

Embora os programas que utilizam estes métodos alcancem os objetivos desejados, tais como a redução do número de medicamentos utilizados, algumas desvan ta gens inclu em o fato de que os revisores não dispõem de orientações preestabelecidas que con duzam o proce sso de revisão, o que faz com que esta ocorra de forma subjetiva, baseada na ex periência clínica do revisor. Ainda que sejam mais próximos à realidade observada no co tidiano da atenção à saúde do idoso, sua validade e confiabilidade são de difícil avaliação.

\section{Métodos explícitos}

Um dos métodos explícitos mais usados na avaliação do uso inadequ ado de medicamentos tem sido o proposto por Beers (Beers et al., 1991; Beers, 1997; Fi cket al., 2003).

Em 1991, Beers et al. criaram o primei ro conjun to de crit é riospara iden tificar o uso inadequado de medicamen tos por idosos institucionalizados nos Estados Unidos. Es te trabalho envo lveu um grupo de especialistas em diferentes áreas do conhecimen to, tais como clínica médica, farmacoepidemiologia, farmacologia geriátrica, entre outros, os quais utilizaram a técnica Delphi. Essa técnica é usada para se obter con s enso a re s pei to de um tema sob inve sti- 
gação. Em geral, os critérios são apresentados aos participantes por meio de inquérito postal. As respostas aos itens são analisadas e um novo questioná rio é el a borado, ac re scen tan do-se ou retiran do-se itens. Os inquéri tos são repetidos até a obtenção de con s enso en tre os participantes (Graham et al., 2003). Por meio desta técnica, estes profissionais definiram, de maneira consensual, uma lista de 19 medicamentos inadequados e 11 medicamentos cuja dose, freqüência de uso ou duração do tratamento eram inadequadas (Beers et al., 1991). Esse critério con templa dois aspectos do uso inadequado de medicamentos por idosos: 1) medicamen tos ou classes de medicamentos que devem ser evitados exceto sob raras circunstâncias e 2) medicamentos cujas doses, freqüências de uso ou duração do tratamen to não devem ser excedidas (Beers et al., 1991). A lista inclui sedativos e hipnóticos, analgésicos, antipsicóticos, antidepressivos, anti-hipertensivos, antiinflamatórios não-esteróides e hipoglicemiantes orais.

Os critérios utilizados nesta época possuíam a va n ta gemde simplificação de uso, uma vez que para sua aplicação são necessárias somente as informações sobre uso de medicamentos disponíveis nas bases de dados. Tendo em vista que estes critérios foram primariamen te direcion ados para idosos institucionalizados, que em geral apresentam piores condições de saúde do que aqueles residen tes na comunidade, os autores ressaltaram a necessidade de modificação da lista nos casos em que esta fosse utilizada para estudos envo lvendo estes últimos. Além disso, salien ta rama importância de atualização da lista, dian tedas constantes alterações no merc ado farm acêutico e da ampliação do conhecimento sobre os fármacos em uso. Salientaram, ainda, a necessidade de expansão dos critérios a fim de que incluíssem variáveis não farmacológicas, tais como diagnósticos, gravid ade das doenças, en tre outros.

Assim, em 1997, Beers atualizou os critérios publicados em 1991 para incluir novos fármacos e incorporar novas evidências da terapia farm acológica. Além disso, o critério foi ampliado para aplicação em idosos não institucionalizados e inclu iu itens adicionais sobre o uso de determinados medicamentos em pacientes com condições patológicas específicas. Esta estratégia resul tou numa lista constituída por 28 medicamentos ou classes de medicamentos inadequados e 35 medicamentos ou classes de medicamen tos considerados inade- quados em 15 condições patológicas específicas (Beers, 1997).

A terceira e mais recen te revisão dos critérios de Beers ocorreu no ano de 2002 (Fick et al. 2003). Algumas diferenças no processo de ssa atualização são bastante interessantes como, por exemplo, o fato de que, para a seleção dos fármacos que iriam compor o questionário a s er res pon di do pelos especialistas, foi utilizada a revisão sistemática. Após esta revisão, foi conduzido um inquéri to dividido em cinco etapas. Finalmente, foram iden tificados, por consenso, 48 medicamentos ou classes de medicamentos in adequ ados e uma lista de medicam en tos inadequ ados em indiví duos com 20 condições pato ló gicas específicas (Fick et al., 2003).

Um outro método baseado em con s enso foi desenvolvido por McLeod et al. (1997) no Canadá. Por meio de um painel com pos to por 32 especialistas, foram identificadas 38 práticas inadequadas de prescrição para idosos. Diferen temen te do critério de Beers et al., estes autores categorizaram as práticas em três tipos: 1) prescrição de fármacos geralmente contraindicados para idosos em função do balanço risco-benefício ser inaceitável; 2) prescrição de fármacos que podem interagir com outros fármacos; 3 ) prescrição de fármacos que podem intera gir com alguma doença. Vale a pena ressaltar que a definição da prim ei ra categoria bas eou-se na lista des envolvida por Beers et al. (1991). Os fárm acos inadequ ados foram agrupados em quatro categorias: cardiovasculares, psicotrópicos, antiinflamatórios não-esteróides e outros analgésicos e fármacos diversos. No entanto as vantagens e desvantagens desse critério são semelhantes àqu elas do crité rio de Beers.

O tercei ro método que utilizoucritérios explícitos é o DUR (Drug Utilization Review), desenvo lvi do por Kna pp em 1989, nos Estados Unidos, e que pode ser usado para avaliar a qualidade e o custo do uso de medicamentos (Knapp, 1991). Geralmente, utilizam-se dados retrospectivos de grandes bancos de dados de prescrições médicas para a identificação de probl emas relaci on ados à do se, du ração do tratamento, duplicação terapêutica e interações medicamentosas (Hanlon et al., 2002). Kn a pp (1991) de s envo lveu crité rios explícitos, baseando-se na literatura científica e no método de con s enso para alguns grupos de medicamentos freqüen temen te usados por idosos e com implicações significativas decorren tes de seu uso indevi do, como antagonistas dos receptores $\mathrm{H}_{2}$ de histamina, digoxina, inibidores da enzima 
conversora de angiotensina, antagonistas dos canais de cálcio, ben zodiazepínicos, antiinflamatórios não-esteróides, antipsicóti cos e antidepressivos. Segundo esse método, por exemplo, o uso prolongado de ben zodiazepínicos e o uso daqueles de longa ação é inapropriado para idosos.

A utilização de critérios explícitos é bastante útil para avaliar o padrão de prescrição ou de uso de medicam en tos por idosos e subsidiar intervenções para otimizar a atenção a esse grupo de indiví duos (Beers et al., 1991). No entanto, algumas limitações devem ser apontadas. Sob circunstâncias específicas, baseadas na avaliação individual, o uso do medicamento considerado inadequado pode ser justificado ( Beers et al., 1991). Dessa forma, não repre sentam contra-indicação absoluta, mas sinalizam que raramente esses medicamentos devem ser utilizados. Outro pon to importante a ser considerado é que os instrum en tos de senvo lvidos a partir deste método não abrangem todos os aspectos relacionados à inadequação, como a subutilização de medicamentos necessários e a administração inadequada (Fick et al., 2003). Além disso, na maioria das vezes estes métodos se restringem à avaliação de fármacos ou grupos de fárm acos específicos.

Uma grande vantagem da utilização dos métodos explícitos é a identificação de grupos vulnerá veis à ocorrência de probl emas relacio$\mathrm{n}$ ados a medicamentos para que sua preven ção seja possível. Os critérios representam, assim, mecanismos para alertar os profissionais de saúde sobre a possibilidade de ocorrência de uso inadequado de medicamentos (Beers, 1997). Além disso, a utilização de listas possibilita a comparação de estudos que avaliaram a adequação do uso de medicam en tos realizados em d iversos países.

\section{Métodos explícitos e implícitos}

Dian te das limitações dos métodos explícitos e implícitos, alguns estudiosos, isoladamente, desenvolveram instrumen tos que combinam critérios explícitose implícitos, com a finalidade de contemplar, de forma mais abrangen te, $o$ e squ ema terapêuti co, a condição clínica geral, bem como circunstâncias especiais do indivíduo, durante o processo avaliativo. Dentre os instrumentos que possuem estas características, os mais importan tes na litera tu ra consultada são aquele desenvolvido por Lipton et al.
(1993) e o MAI (Medication Appropriateness In dex), criado por Ha nlonet al. (1992).

\section{Método de Lipton}

O de s envo lvimen to des te instru m en to caracterizou uma etapa de um ensaio dínicorealizado nos Estados Unidos, em 1989, com o obj etivo de avaliar o impacto da participação de farmacêuti cos díni cos no processo da prescrição médica para 236 pacien tes hospitalizados com mais de 60 anos (Lipton et al., 1993).

O con teú do do instrumen to foi estabelecido por meio da realização de um painel form ado por cinco clínicos e dois farmacêuti cos clínicos. Foram definidas seis categorias de problemas de prescrição. Além disso, foi cri ado um sistema de escores variando de 0 a 2 , p a ra dassificação da magnitude do problema de cada c a tegoria (Quad ro 1). A partir dos escores parciais, foi calculado um escore gl obal de inadequação da pre s c rição para cada pacien te.

Pa ra o processo de avaliação da farm acoterapia, os participantes do painel utilizaram os registros médicos, fon tes de informação sobre interações medicamentosas e informações sobre o utros fármacos em uso pelos pacientes (obtidas por meio de en trevistas telefônicas). Para cada caso (paci en te ), a avaliação foi re a li z ada por um par de membros do painel, de forma duplo-cega.

Foi realizado te ste de confiabilidade do instrumen to, a partir do número de escores discrepantes, durante os seis primei ros meses das revisões. Observou-se que a discrepância diminuiu substancialmente ao longo do tempo. A análise da validade ex terna do instrumento não foi possível em função da inexistência de um padrão-ourode comparação. En tretanto, foi re alizada uma análise indireta buscando-se uma relação en tre os escores médios de prescrição e o relato de reações adversas pelos pacientes e, ainda, o número de admissões hospitalares relacion adas com o uso de medicamentos. Os autores evidenciaram um percen tual discretam en te superior $(7,0 \%)$ de hospitalizações en tre aqueles pacien tes com pelo menos um problema potencialmen te fatal $(n=52)$ comparado ao percen tual de $4 \%$ de hospitalização para os demais $(n=184)$. Observou-se ainda uma correlação po si tiva en tre o escore global de inadequação e o número de reações adversas rel a tadas (Lipton et al., 1993).

O método de Lipton apresenta algumas vantagens, como a existência de crit érios explí- 
Quadro 1

Categorias de probl emas de pre s c rição e escores de classificação - critério de Lipton.

\begin{tabular}{|c|c|}
\hline Ca tegoria de problema & Es core de dassificação \\
\hline 1. Hipersensibilidade medicamentosa & 0. Não há problema \\
\hline 2. Dose do fármaco & $\begin{array}{l}\text { 1. Si tuação provavelmen te inadequ ada (dinicamente significativa, } \\
\text { mas não fatal) }\end{array}$ \\
\hline 3. Freqüência de administração do fárm aco & $\begin{array}{l}\text { 2. Si tuação definidamente inadequ ada (potencialmente fatal ou } \\
\text { com po tencial para re sultar em prejuí zos graves/ hospitalização) }\end{array}$ \\
\hline 4. Contra-indicação/indicação do fárm aco & 9. In formação insuficiente para avaliar a situação \\
\hline \multicolumn{2}{|l|}{ 5. In teração medicamentosa } \\
\hline 6. Duplicidade farmaco terapêutica & \\
\hline
\end{tabular}

citos combinados com o julgamen to implícito do avaliador. Outra vantagem é o fato de que o instrumento não é fármaco-específico, como ocorre com o DUR. Ao contrário, ele permite uma avaliação do fárm aco no con tex to da condição clínica de cada indivíduo. En tretan to, algumas desvantagens também devem ser apontadas. Uma vez que cada fárm aco é avaliado ind ividualmente, a aplicação do instrumen to pode ser demorad a. Outro aspecto destac ado pelos autores como desvantagem do instrum en to é a estreita faixa dos escores. O aumento da amplitude da faixa permitiria maior sensibilidade do instrumen to à gravidade dos probl emas de prescrição avaliados (Lipton et al., 1993). Por fim, são necessárias novas análises de confiabilidade e validade do instrumento para melhor avaliação de s te aspecto e expansão da aplicação de s te instrumen to (Shel ton et al., 2000).

\section{Medication App ropria teness Index (MAI)}

O MAI é um instrumento bastante semelhante ao de senvolvido por Lipton et al. e também foi el a borado como parte de um ensaio dínicopara avaliação de serviços em saúde nos Estados Unidos, desenvolvido por Hanlon e colaboradores no início da década de 1990 (Hanlon et al., 1992). Di feren temen te do método desenvolvido por Lipton e colaboradores, este instru men to é mais abra nen te e já foi submetido a diversos testes de confiabilidade e validade (Hanlon et al., 1992; Samsa et al., 1994; Fitzgerald et al., 1997).

O instrumento foi el a borado por um geri atra e um farmacêutico dínico e consta da ava- liação de 10 elemen tos da prescrição medicamentosa. Pa ra cada el em en to foram estabelecidas definições operaci onais e instruções para a avaliação. O índice foi desenvo lvi do a partir de uma escala de três pontos, em que os índices 1 , 2 e 3 representam, respectivamente, "uso adequ ado", "uso marginalmen te adequado" e "uso in adequ ado" (Qu ad ro2).

Outra etapa no desenvolvimen to do MAI foi a definição e validação de um processo de ponderação no qual cada uma das dez categorias foi pontuada a fim de se obter um escore global por medicamento. A pon tuação variava de 1 a 5 , de forma que um escore igual a 1 para aqu ela categoria signific avaausência de importância, ao passo que um escore de 5 atribuía gra n de importância para a categoria. Pa ra isso, dois farmacêuti cos clínicos avaliaram, individualmente, um total de 105 fármacos prescritos para dez idosos hospitalizados numa clínica de medicina interna nos Estados Unidos. Nesta avaliação o escore gl obal apre s en tou uma confiabilidade aceitável (coeficien te de correl ação intraclasse igual a 0,74$)$. Esse procedimento aponta para a validade de con teú do do instrumen to (Samsa et al.,1994).

O instrumen to foi ainda submetido à análise de confiabilidade, sen do os coeficientes Kappa obti dos (variação de 0,54 a 0,92) considerados satisfatórios para que o seu uso seja recom en dado em populações de idosos não hospitalizados (Hanlon et al. 1992; Fitzgerald et al., 1997).

De forma semelhante ao método de Lipton, o método MAI não teve sua validade ex terna atestada, devido à ausência de um padrão de comparação. Por outro lado, foi ava li adaa relação entre o escore global de cada fármaco e a 
Quadro 2

Ca tegorias de probl emas de prescrição e escores de classificação - Medication Appropriateness In dex.

\begin{tabular}{|c|c|}
\hline Ca tegoria de problema & Escore de dassificação \\
\hline 1. Indicação do fármaco & $\begin{array}{l}\text { 1. Indicado; 2. Marginalmen te indicado; } 3 \text {. Não indicado; } \\
\text { 9. Não é possível avaliar }\end{array}$ \\
\hline 2. Efetividade do fárm aco para a condição & $\begin{array}{l}\text { 1. Efetivo; } 2 . \text { Marginalmente efetivo; } 3 \text {. In efetivo; } \\
\text { 9. Não é possível avaliar }\end{array}$ \\
\hline 3. Adequação da dose & $\begin{array}{l}\text { 1. Adequada; } 2 \text {. Marginalmente adequad a; } 3 \text {. In adequada; } \\
\text { 9. Não é possível avaliar }\end{array}$ \\
\hline 4. Ori entação ao pacien te para o uso do fárm aco & $\begin{array}{l}\text { 1. Correta; } 2 \text {. Marginalmente correta; } 3 \text {. Incorreta; } \\
\text { 9. Não é possível avaliar }\end{array}$ \\
\hline 5. Conveniência & $\begin{array}{l}\text { 1. Correta; } 2 \text {. Marginalmente correta; } 3 \text {. Incorreta; } \\
\text { 9. Não é possível avaliar }\end{array}$ \\
\hline 6. In terações medicamentosas & $\begin{array}{l}\text { 1. Insignificantes; } 2 \text {. Marginalmente significantes; } 3 \text {. Significantes; } \\
\text { 9. Não é possível avaliar }\end{array}$ \\
\hline 7. In terações fármaco - doença/ condição dínica & $\begin{array}{l}\text { 1. Insignificantes; } 2 \text {. Marginalmente significantes; } 3 \text {. Significantes; } \\
\text { 9. Não é possível avaliar }\end{array}$ \\
\hline 8. Duplicação farmaco terapêutica & $\begin{array}{l}\text { 1. Necessária; } 2 \text {. Marginalmente necessária; } 3 \text {. Desnecessária; } \\
\text { 9. Não é possível avaliar }\end{array}$ \\
\hline 9. Duração do tratamen to & $\begin{array}{l}\text { 1. Aceitável; 2. Marginalmente aceitável; } 3 \text {. In aceitável; } \\
\text { 9. Não é possível avaliar }\end{array}$ \\
\hline $\begin{array}{l}\text { 10. Cu s to do fárm aco em comparação a outro(s) } \\
\text { de seg u rança e eficácia semelhantes }\end{array}$ & $\begin{array}{l}\text { 1. Mais barato; 2. Custo mediano 3. Mais caro; } \\
\text { 9. Não é possível avaliar }\end{array}$ \\
\hline
\end{tabular}

utilização de serviços por idosos. Observou-se que a média do escore era superior (ou seja, uso mais inadequ ado) para aqueles indivíduos com maior número de hospitalizações (Sch mader et al., 1997).

É interessante ressaltar que ambos os métodos, de Lipton e o MAI, não avaliam reações adversas a medicamentos (RAM) de uma maneira global. No MAI, somen te as interações medicamen tosas são con tem pladas e no método de Lipton, além de interações, a hipersensibilidade é considerada no processo de avaliação. No que se refere ao MAI, os autores atribu em a ausência desse critério no instrumen to à complexidade que envo lve a determinação da ca us alid adedeuma RAM, bem como à existência de algori tmos pró prios para essa finalidade (Hanlon et al., 1992).

$\mathrm{Na}$ atualidade, é possível que o MAI seja o instrumen to mais completo, válido e reprodutível dispon ível para avaliar a adequação da terapia farmacológica em idosos. Sua principal desvantagem é o tempo requerido para completar a revisão de cada medicamento, o que é estimado em 10 minutos. Por outro lado, se utilizado de acordo com as definições opera- cionais, torna-se uma ferram enta estrutu rada e sistemática de revisão, ao mesmo tempo em que confere uma flexibilidade relacionada à particularidades de cada indivíduo em uso de medicamento.

\section{Avaliação da qualidade do uso de medicamen tos por idosos no Brasil}

Os idosos são o grupo etário que cresce mais rapidamente no Brasil. A demanda deste grupo por recursos de saúde é intensa, tanto no que se refere à utilização de serviços de saúde quan to no que diz re s pei to ao uso de medicamentos.

Entretanto, no campo da farmaco terapia, o cenário que se vislumbra é do dilema "acesso ou excesso". Este dilema é repre sen tado pela dificuldade na obtenção de medicamentos, pela subutilização de classes terapêuticas específicas, pelo con sumo irraci onal de novidades químicas (Rozen feld, 2003), enfim, por distorções nos diferentes elementos que compõem a cadeia medicamentosa. A coexistência dessas distorções propicia, indubit avelmente, o uso inadequ ado de medicamen tos por idosos brasilei 
ros. Esta situação carece de iniciativas e mesmo de uma discussão mais aprofundada para seu adequ ado en frentamento.

Nesse con texto, tão importante quan to o conhecimento dos diferentes métodos utilizados para avaliar a adequação da farmacoterapia em idosos é se vislumbrar o potencial de uso dessas ferramentas diante das particularid ades dos serviços de saúde no Brasil.

A partir da revisão apresentada, é possível observar que a grande maioria dos métodos é aplicada utilizando-se informações sobre o uso de medicamen tos obtidas em prescrições médicas, bases de dados de farmácias e/ou registros médicos. É perceptível que a qualidade dessas fon tes é fundamental para que o uso desses instrumentos produza resultados compatíveis com a realidade e, em especial, úteis como alvos de estratégias de intervenção. Esta condição é um primei ro desafio para a transposição destes métodos para o Brasil. Aqui, fontes como os bancos de dados dos programas de a tenção à saúde ou das contas hospitalare s, em geral não dispõem de registros sobre o uso de medicamentos. Eventualmente, os programas de assistência farmacêutica em sistemas locais de saúde possu em informações sobre a distribuição de fárm acos, mas é incomum en contrar as variáveis representativas do uso de medicamentos associadas às variáveis de morbidade ou socioeconômicas, de forma que se possam avaliar, sistematicamen te e em profundidade, os diferen tes aspectos do processo de utilização dos mesmos. Além disso, o preen chimen to dos prontuários médicos em diferentes níveis de atenção à saúde é de qualidade bastante insatisfatória, em especial no que tange às inform ações relacionadas aos medicamen tos prescritos. A adoção de estratégias foc adas na qualidade das informações sobre o uso de medicamentos nas diversas fon tes mencionadas é de grande valia para a implem entação de programas de avaliação da farm acoterapia para pessoas com 60 anos ou mais.

Num segundo mom en to, ao se con s i derar a possibilidade da utilização de listas de medicamen tos classificados como inadequados, é fundamental ressaltar a necessidade de adaptação destas listas à realidade do merc ado farmacêutico brasileiro. Isso porque alguns fárm acos comercializados nos países onde as listas foram desenvolvidas não o são aqui e algumas especialidades utilizadas aqui não o são lá. Ne s te caso, o mais coeren te é que sejam elaboradas listas próprias, ou seja, que traduzam a realidade brasileira. Estas listas, obtidas por consen so, poderiam ser úteis às atividades de seleção de medicamentos, bem como poderiam subsidiar a elaboração de protocolos terapêuticos que con tem plem as particularid ades dos indivídu os com mais de 60 anos. Os centros colaboradore s de Geriatria e Geron tologia do país, as universidades abertas da tercei ra idade, jun tam en teàs comissões de pad ronização de medicam en tos e aos órgãos reguladores, ressalt ado o seu caráter multidisciplinar, podem exercer um importante papel neste processo, dandosu porte ou mesmo des en cade an do ações neste sen tido.

Outro aspecto de importância no que se refere à utilização dos métodos apres en tados para avaliar a adequação da farmaco terapia em idosos no Brasil é a evidência de um espaço que requ er a atuação do farmacêutico. Em geral, todos os métodos discuti dos aqui foram el a borados e/ou conduzidos com a participação do farmacêuti co. Além disso, é interessan teob servar que os instrumentos utilizados pelos métodos implícitos e por aqueles que con ju gam critérios explícitos e implícitos se aproximam mu i to da prá tica da Atenção Fa rmacêutica. Esta, por sua vez, tem como um dos seus obj etivos, garantir que os medicamentos utilizados sejam indicados, efetivos, seguros e convenientes, maximizando os ben eficios e minimizando os riscos decorrentes de seu uso (Cipolle et al., 1998).

\section{Considerações finais}

Discutir a qualidade da farm aco terapia em idosos é discutir a atenção à sua saúde, tendo em vista que o medicamento ainda é um importante instrumen to de recuperação e manutenção da saúde de indivíduos des te gru po populacional, embora resguardada a preocupação com a não medicalização do con cei to de saúde. Assim, a avaliação da farmacoterapia em idosos é um importante instrumen to de avaliação da qualidade da atenção prest ada a este gru po. E s forços para aprimorar a seleção, prescrição, a dispensação e a utilização de fárm acos devem constituir prioridade nos programas de atenção ao idoso.

O presente artigo apresen tou diferentes métodos utilizados para a avaliação da qualidade do uso de medicamen tos em idosos, com o intui to de subsidiar a discussão em torno da Farm acoepidemiologia do envelhecimento no país. Embora se trate de um artigo de revisão, diante da complexidade que permeia os pro- 
cessos de "qualidade", "uso de medicamentos"e "envelhecimen to populacional”, é importante ressaltar que os métodos apre sen tados não preten dem esgotar as possibilidades de avaliação e intervenção nesta temática. Não obstante, a

\section{Colaboradores}

AQ Ribeiro e CMCA realizaram a busca na base de dados, a seleção e análise dos documentos e a redação do artigo. FA Acurcio, SMS Ma galhães e F Ch a i m owicz participaram da análise dos documentos. Todos os autores tom a ram parte na el a boração do arti go final.

\section{Referências bibli ográficas}

Beers MH et al. 1991. Explicit cri teria for determining inappropriate medication use in nursing home residents. Arch ives of Internal Medicine 151:1825-1832.

Beers MH 1997. Explicit cri teria for determining po tentially inappropria temedication use by the el derly: an update. Arch ives of Internal Medicine 157:1531-1536.

Chaimowicz F 1997. A saúde dos idosos brasileiros às vésperas do século XXI: problemas, projeções e alternativas. Revista de Saúde Pública 31(2):184-200.

Chaim owicz F, Ferreira TJXM \& Miguel DFA 2000. Use of psych oactive drugs and related falls among older people living in a community in Brazil. Revista de Saúde Pública 34(6):631-635.

Cipolle RJ, Strand LM \& Morley PC 1998. Pharmaceutical care practice. Mc Graw Hill, New York.

Coelho Filho JM, Ma rcopito LF \& Ca s telo A 2004. Perfil de utilização de medicamentos por idosos em área urbana do Nordeste do Brasil. Revista de Saúde Pública 38(4):557-564.

Fick DM et al. 2003. Updating the Beers criteria for potentially inappropria temedicati on use in older adults: re sults of a US consensus panel of experts. Archives of In ternal Medicine 163(22):2716-2724.

Fitzgerald LS et al. 1997. Reliability of a modified medication appropriateness index in ambula tory older pers on s. The Annals of Pharmacotherapy 31:543-548.

Graham B, Regehr G \& Wright JG 2003. Delphi as a method to establish consen sus for diagnostic criteria. Journal of Clinical Epidemiology 56:1150-1156.

Ha $\mathrm{l}$ on JT et al. 1992. A met h od for assessing drug therapy appropriateness. Journal of Clinical Epidemiology 45(10):1045-1051.

Hanlon JT et al. 2002. Use of inappropriate prescribing dru gs by older people. Journal of the American Geria tri cs Society 50(1):26-34.

Katzung BG 2002. Aspectos especiais da farmacologia geriátrica, pp. 899-906. In BG Katzung BG (org.). Farmacologia básica \& dínica. 8a ed. Ed. Guanabara Koogan, Rio de Ja neiro.

Knapp DA 1991. Devel opment of criteria for drug utilization revi ew. Clinical Pharmacology and Therapeutics 50(5):600-602.

Last JM 1989. Dicionário de Epidemiologia. Ed. Salvat, Barcelona. p. 54. adequação ou adaptação destes métodos à re alidade brasileira e a incorporação dos mesmos às práticas avaliativas em saúde pode se con stituir em um passo fundamental na promoção do uso raci onal de medica men tos no país.
Lipton HL, Bird JA, Bero LA \& McPhee SJ 1993. Assessing the appropri a teness of physician prescri bing for geria tric outpatients: developm ent and testing of an instrument. Journal of Pharmacy Technology 9:107-113.

Mc Leod PJ, Huang AR, Tamblyn RM \& Gayton DC 1997. Defining inappropriate practices in prescribing for el derly people: a nati onal con s en sus panel. Canadian Medical Association Journal 153(3):385-391.

Miralles MA \& Kimberlin CL 1998. Perceived access to care and medicati on use among ambula tory el derly in Rio de Janei ro, Brazil. Social Science \& Medicine 46(3):345-355.

Mosegui GBG, Rozenfeld S, Veras RP \& Vianna CMM 1999. Avaliação da quali da de do uso de medicamentos em idosos. Revista de Saúde Pública 33(35):437444.

Rozen feld S 1997. Reações adversas aos medicamentos na terceira idade: as quedas em mu l h eres como iatro genia farmacoterapêtica. Tese de doutorado. Instituto de Medicina Social, Un iversidade Estadual do Rio de Jan eiro, Rio de Jan ei ro.

Rozen feld S 2003. Prevalência, fa tores associados e mau uso de medicamentos entre idosos: uma revisão. Cademos de Saúde Pública 19(3):717-724.

Samsa GP et al. 1994. A su m mated score for the medicati on appropri a teness index: devel opm ent and assessment of dinimetric properties including content validity. Journal of Clinical Epidemiology 47(8):891-896.

Schmader KE et al. 1997. Inappropria te prescribing and health outcomes in el derly veteran outpatients. The Annals of Pharmacotherapy 31:529-533.

Schrader SL et al. 1996. The medication redu ction project: combating polypharm acy in So uth Dakota elders thro u gh com mu nity-b a s ed interven tions. South Dakota Journal of Medicine 49(12):441-448.

Shel ton OS, Fritsch MA \& Scott MA 2000. Assessing medication appropriateness in the elderly: a review of available measu res. Drugs \& Aging 16(6):437-450.

Siquei ra RL, Bo telho MIV \& Coelho MG 2002. A velhice: algumas considerações teó ricas e con ceituais. Ciência \& Saúde Coletiva 7(4):899-906.

Artigo apresentado em 26/11/2004

Aprovado em 18/04/2005

Versão final apresentada em 18/04/2005 